\title{
Sarcoidosis Associated With Electronic Cigarette Use in an Adult: A Case Report
}

\author{
Alexandria Soybel $^{\mathrm{a}, \mathrm{b}}$, Victoria DeJaco ${ }^{\mathrm{b}}$, Alejandra Ellison-Barnes ${ }^{\mathrm{b}, \mathrm{c}}$, \\ Panagis Galiatsatos ${ }^{a, b, d}$
}

\begin{abstract}
The clinical recognition of acute lung disease caused by electronic cigarette (e-cigarette) usage has continued to grow. The identified pulmonary pathologies each include some degree of injury and/or inflammation. Electronic cigarettes contain chemicals that hold potential for acute pulmonary toxicity, including, but not limited to, nicotine, volatile organic compounds, and aldehydes. However, the development of chronic pulmonary diseases with more insidious symptoms has gained more recognition. Some chronic pulmonary diseases, such as sarcoidosis, have not yet been causally linked to electronic cigarette use. We present a case of a patient who developed sarcoidosis after using electronic cigarettes, discussing clinical outcomes and management of both the patient's electronic cigarette addiction and sarcoidosis development.
\end{abstract}

Keywords: Electronic cigarette; Sarcoidosis; Pulmonary disease; Tobacco use

\section{Introduction}

The clinical understanding of acute lung disease due to electronic cigarettes (e-cigarettes) has been well established over the last decade. The identified pulmonary pathologies each include some degree of injury and/or inflammation, ranging from lipoid pneumonia to hypersensitivity pneumonitis to acute respiratory distress syndrome [1-4]. Electronic cigarettes contain chemicals that hold potential for acute pulmonary toxicity: nicotine, volatile organic compounds, and aldehydes [5].

Manuscript submitted December 21, 2021, accepted February 9, 2022

Published online March 5, 2022

a Division of Pulmonary and Critical Care Medicine, Johns Hopkins School of Medicine, Baltimore, MD, USA

${ }^{b}$ The Tobacco Treatment and Cancer Screening Clinic at the Johns Hopkins Medicine, Baltimore, MD, USA

'Division of General Internal Medicine, Johns Hopkins School of Medicine, Baltimore, MD, USA

${ }^{\mathrm{d} C o r r e s p o n d i n g ~ A u t h o r: ~ P a n a g i s ~ G a l i a t s a t o s, ~ D i v i s i o n ~ o f ~ P u l m o n a r y ~ a n d ~ C r i t i-~}$ cal Care Medicine, Johns Hopkins School of Medicine, Baltimore, MD 21287, USA. Email: panagis@jhmi.edu

doi: https://doi.org/10.14740/jmc3887
In addition to these known active chemicals, their mixing with various contaminants likely results in new chemicals with their own toxicities [6]. However, the development of chronic pulmonary disease with more insidious symptoms is gaining more attention given the growth of electronic cigarette usage and users, both in chronicity and frequency.

Sarcoidosis is a systemic inflammatory disease of unknown cause; and while it may affect any organ, the majority of patients with sarcoidosis have pulmonary involvement [7]. Diagnosing pulmonary involvement of sarcoidosis takes into account a patient's symptoms, clinical imaging (hilar lymphadenopathy and pulmonary nodules), along with a biopsy of the suspicious pulmonary findings. The histological finding of sarcoidosis is that of non-caseating granulomas, which if found, prompts an investigation of other causes of granulomatous disease [7, 8]. To date, sarcoidosis' association with electronic cigarette has been associated with exacerbations of the underlining disease [9], with no association as a potential causal exposure of the development of the systemic disease itself.

We present a case of a patient who developed sarcoidosis after using electronic cigarettes. In the case presentation, we aim to present evidence for electronic cigarette use as a potential cause of the development of sarcoidosis, as well as a potential cause of disease progression.

\section{Case Report}

\section{Investigations}

Our patient is a 50-year-old white female with a history of mild, persistent asthma and tobacco dependence who presented to clinic to discuss new onset dyspnea in the setting of electronic cigarette usage. Her asthma dates back to her childhood, with no active issues as an adult. She only uses a rescue inhaler as needed (per patient, about once a month). The patient began to smoke combustible cigarettes at the age of 18 , smoking about 20 cigarettes a day until the age of 49 , when she switched to electronic cigarettes as recommended by a peer. However, the patient found the electronic cigarette addiction more aggressive and expensive, going through two to three pods a day. This motivated her to seek counseling and guidance on her electronic cigarette addiction. During the clinic visit, electronic cigarette addiction management included counseling, motivational interviewing, and transdermal nicotine replacement therapy [10]. 

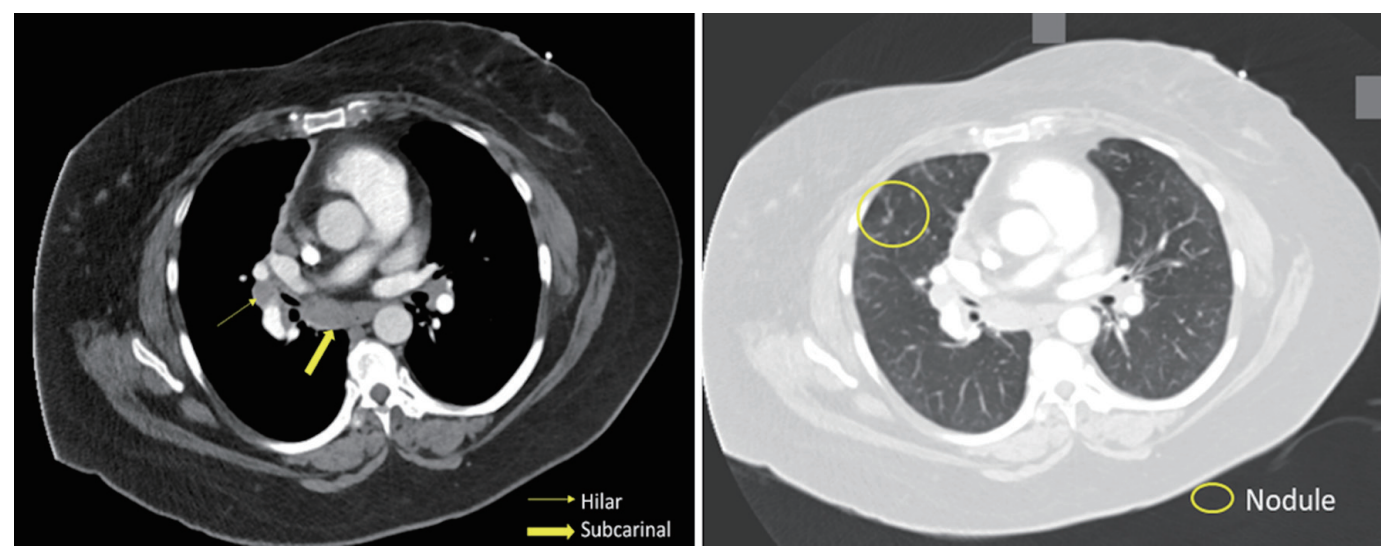

Figure 1. Patient's CT scan of the chest revealing hilar lymphadenopathy, pulmonary nodules, and ground-glass opacifications. CT: computed tomography.

As for the patient's dyspnea, a resting pulse oximeter found the patient's oxygen saturation at $94 \%$; with ambulation, the patient's oxygen saturation levels fell to $80 \%$ after 1-min of walking, necessitating $2 \mathrm{~L}$ of nasal cannula to rebound to a pulse oximeter reading of $96 \%$. Given this finding, the patient was discharged from the clinic with supplemental oxygen and sent to the emergency department for an acute workup.

In the emergency department, the patient's laboratory data, including a complete blood count (CBC) and a complete metabolic panel, were unremarkable. No significant elevations were seen in eosinophils, neutrophils, or lymphocytes in the CBC. The patient's C-reactive protein was undetectable. On computed tomography angiography (CTA) of the chest, there was no evidence of a pulmonary embolism; however, diffuse ground-glass opacities, scattered pulmonary nodules, and hilar $(1.8 \mathrm{~cm})$ and subcarinal $(1.7 \mathrm{~cm}$ ) lymphadenopathy were found (Fig. 1). Of note, in a prior CTA of the chest 3 years prior (obtained due to a motor vehicle accident), there were no signs of lymphadenopathy or ground-glass opacities. The patient was admitted to the hospital for further evaluation. After initiation of antibiotics and a 3-day hospitalization for presumed community-acquired pneumonia, the patient was discharged home to complete the course. Oxygen was still required with ambulation.

The patient's dyspnea continued after the completion of the antibiotics. At a follow-up clinic visit 3 months after hospitalization, a pulse oximetry evaluation now found the patient warranted $2 \mathrm{~L}$ oxygen via nasal cannula at rest, in addition to $3 \mathrm{~L}$ with mobility. A repeat CTA of the chest performed the same day demonstrated persistent lymphadenopathy and ground-glass opacities. The patient was scheduled for an endobronchial ultrasound bronchoscopy with a bronchoalveolar lavage (BAL). From the lavage, no microbial findings were identified. A cell count demonstrated a white blood cell count of $123 / \mathrm{mm}^{3}$, with the differential at $43 \%$ lymphocytes (and an elevated cluster of differentiation (CD)4/CD8 ratio), 22\% polymorphonuclear leukocytes, $35 \%$ monocytes, and $0 \%$ eosinophils. On biopsy of the right hilar and subcarinal lymph nodes, non-necrotizing granulomatous inflammation was found on a background of scattered lymphocytes and benign respiratory epithelium (Fig. 2). No malignant neoplasm was identified.

An investigation was initiated to identify a potential cause of the non-necrotizing granulomatous inflammation. An autoimmune panel was sent; anti-neutrophil cytoplasmic (ANCA) antibody, anti-La antibody, anti-Ro antibody, anti-doublestranded DNA antibody, anti-nuclear antibody (ANA), antiglomerular basement membrane antibody, anti-phospholipid antibody, anti-Smith and anti-ribonucleoprotein (RNP) antibodies were all negative. Fungal serologies for histoplasmosis were negative, as was a hypersensitivity panel to common pathogens.

\section{Diagnosis}

With the extensive differential for pulmonary non-necrotizing granulomatous inflammation, after the laboratory data returned negative, the diagnosis of sarcoidosis was considered. Several findings made sarcoidosis likely. First, the finding of lung nodularity on imaging was suspicious. Pulmonary nodules in sarcoidosis represent aggregation of granulomas and can be found up in greater than $80 \%$ of patients with sarcoidosis [11]. And the pulmonary nodules were found alongside the mosaic attenuation, seen with extensive ground-glass opacities in our patient (Fig. 1) [11]. Second, mediastinal and hilar lymphadenopathy is

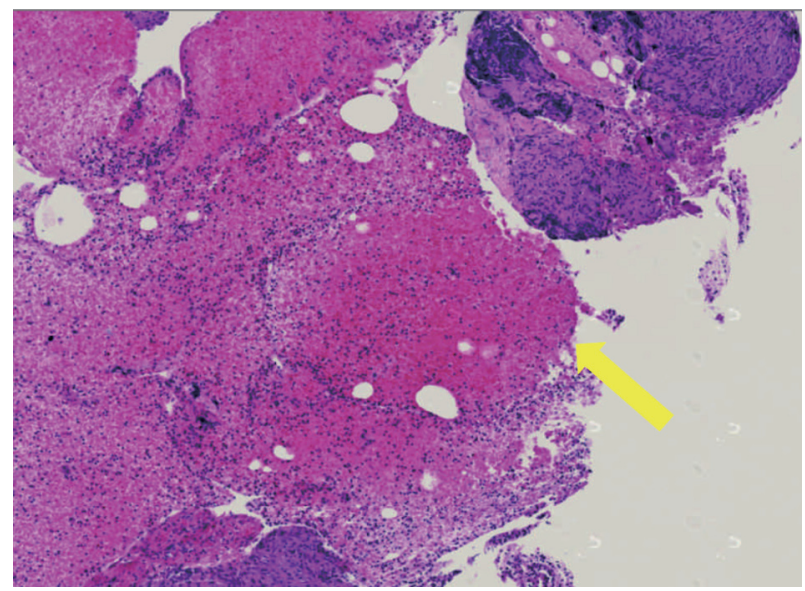

Figure 2. Histopathological slide of patient's pulmonary lymph node biopsy revealing non-caseating granulomas (yellow arrow). 
well documented in sarcoidosis [11], and was present extensively in our patient. Finally, the high lymphocytes and elevated CD4/ CD8 ratio seen in the BAL is commonly seen in sarcoidosis [12]. Therefore, considering the patient's pathological findings, cell count findings on BAL, and imaging, sarcoidosis was diagnosed.

\section{Treatment}

After 8 months of steroids for pulmonary sarcoidosis (starting at prednisone $20 \mathrm{mg}$ daily and tapering to a prednisone $5 \mathrm{mg}$ daily), as well as successfully weaning and ultimately stopping her electronic cigarette usage, the patient was able to come off of oxygen, and her dyspnea resolved. The patient felt that her endurance was back to baseline. Repeat imaging revealed a resolution of the pulmonary nodules and the ground-glass opacities, and a reduction in size of the mediastinal and hilar lymphadenopathy. Of note, pulmonary function testing was recommended but not performed due to scheduling conflicts. The patient continued to receive counseling for electronic cigarette abstinence while on $5 \mathrm{mg}$ of prednisone.

\section{Follow-up and outcomes}

At the age of 52 years, the patient had a relapse of her electronic cigarette usage. During this relapse, the patient developed dyspnea over a 1-month course, again resulting in the need for oxygen. Upon hospitalization for worsening dyspnea, repeat imaging showed a return of pulmonary nodules and ground-glass opacities. The patient required invasive mechanical ventilation due to hypoxemic respiratory failure, and on hospital day 4 she had a cardiac arrest in the intensive care unit and passed away.

Of note, there was a strong suspicion that the electronic cigarette use resulted in the patient's sarcoidosis trajectory. The patient developed sarcoidosis findings and symptoms 10 months after initiating electronic cigarette usage. The patient's sarcoidosis symptoms and imaging findings both seem to have been mitigated and resolved, respectively, with abstinence from electronic cigarette usage in tandem with prednisone (main therapy for sarcoidosis). She developed a recurrence of symptom and imaging findings of sarcoidosis after relapse of electronic cigarette usage. Therefore, the patient's sarcoidosis may have been caused and influenced by electronic cigarette usage.

\section{Discussion}

Sarcoidosis is a multisystem inflammatory disease with a wide range of clinical manifestations, characterized by the formation of non-caseating granulomas. Sarcoidosis is thought to represent an abnormal immune response to an antigen exposure, with both genetic and environmental factors playing a role in the onset of disease as well as clinical expression. In our case presentation, we describe the clinical course of incident sarcoidosis developing after electronic cigarette initiation, resolution of symptoms with abstinence of electronic cigarette usage, and return of symptoms with relapse of electronic cigarette usage.
The triggering antigen or antigens for the pathogenesis of sarcoidosis is likely to vary according to environment (geographic location) and individual (race, genetics, ethnicity) factors [13]. With such insight, electronic cigarettes may result in an aberrant immune reaction that may result in sarcoidosis. The chemicals involved in electronic cigarettes may have significant antigenic properties. For example, isocyanates may combine with host proteins to form haptens, which in turn are antigenic, with such a property being described to play a role in sarcoidosis pathogenesis [14]. Various aerosolized chemical substances in electronic cigarettes can react with human proteins and induce an exaggerated immune response in susceptible persons, a phenomenon that has been well-characterized in dust and smoke aerosols [15]. Further, the chemicals of electronic cigarettes may react to create other novel chemicals that may in turn trigger an exaggerated immune response.

It should also be noted that electronic cigarettes hold potential for the growth of microbial antigens, specifically mycobacteria. Metalworking fluids, a known component of electronic cigarettes, may contain carbon and water and have been identified to sustain growth of microbes, specifically mycobacteria [16]. Such bacteria are well known to grow in high temperatures and within aerosols of water droplets, making it plausible that they may be in electronic cigarette aerosols. Further, mycobacteria are often discussed as potential causes of sarcoidosis [16]. This is suspected because mycobacteria are slow-growing microbes with low pathogenic potential and significant capability to elicit a type IV immune response [16]. Therefore, both the chemicals and potential microbes that have been identified in electronic cigarettes hold anergic capabilities that may result in sarcoidosis.

The patient's pulmonary sarcoidosis was managed with standard glucocorticoid therapy. However, the patient in parallel was also weaned off electronic cigarettes. When she relapsed to electronic cigarette usage, she saw an aggressive return of her sarcoidosis, necessitating hospitalization and critical care resources, and ultimately resulting in respiratory failure and death. Therefore, for patients who are using electronic cigarettes and are identified to have a non-caseating granulomatous disease, such as sarcoidosis, disease management should also include abstinence from electronic cigarettes.

In conclusion, our case demonstrates the potential development of incidental sarcoidosis in an adult female due to electronic cigarette usage. Her disease severity paralleled her electronic cigarette usage, with resolution of sarcoid-related symptoms and signs during her abstinent period, and a return of aggressive clinical manifestations during her relapse to electronic cigarette use. More information is needed to understand the composition of the aerosols generated by electronic cigarettes, their antigenic potential, and their plausibility in resulting in granulomatous diseases, such as sarcoidosis. Our case should prompt caution with electronic cigarette usage given they are a potential etiology for pulmonary diseases resulting from aberrant and/or exaggerated immune responses.

\section{Learning points}

Electronic cigarettes and their impact on pulmonary health continue to be explored, with ongoing investigations into the 
pathogenesis of certain diseases impacted by electronic cigarette usage. Assuring a documentation of the usage of electronic cigarettes and its potential correlation, association, and/or causation of an established pulmonary illness should be on the minds of all healthcare professionals. In the case of our patient, such insight was necessary for the treatment of her sarcoidosis, while her relapse in the usage of the electronic cigarette resulting in worsening of her pulmonary symptoms and disease.

\section{Acknowledgments}

None to declare.

\section{Financial Disclosure}

The authors have no financial disclosure.

\section{Conflict of Interest}

The authors declare they have no conflict of interest.

\section{Informed Consent}

Informed consent was obtained by the patient's next of kin for this presentation.

\section{Author Contributions}

AS performed the necessary background investigation, summarizing all references and discussion points. AEB and VD assisted in the write-up of the manuscript. PG oversaw the genesis of the manuscript and editing, cared for the patient and provided the case summary. All authors contributed to editing the manuscript.

\section{Data Availability}

The data supporting the findings of this study are available from the corresponding author upon reasonable request.

\section{References}

1. Layden JE, Ghinai I, Pray I, Kimball A, Layer M, Tenforde MW, Navon L, et al. Pulmonary illness related to E-cigarette use in Illinois and Wisconsin - final report. N Engl J Med. 2020;382(10):903-916.

2. Xie W, Kathuria H, Galiatsatos P, Blaha MJ, Hamburg NM, Robertson RM, Bhatnagar A, et al. Association of electronic cigarette use with incident respiratory condi- tions among US adults from 2013 to 2018. JAMA Netw Open. 2020;3(11):e2020816.

3. Sommerfeld CG, Weiner DJ, Nowalk A, Larkin A. Hypersensitivity pneumonitis and acute respiratory distress syndrome from E-cigarette use. Pediatrics. 2018;141(6):e20163927.

4. Werner AK, Koumans EH, Chatham-Stephens K, Salvatore PP, Armatas C, Byers P, Clark CR, et al. Hospitalizations and deaths associated with EVALI. N Engl J Med. 2020;382(17):1589-1598.

5. Klager S, Vallarino J, MacNaughton P, Christiani DC, Lu Q, Allen JG. Flavoring chemicals and aldehydes in E-cigarette emissions. Environ Sci Technol. 2017; 51(18):10806-10813.

6. Krishnasamy VP, Hallowell BD, Ko JY, Board A, Hartnett KP, Salvatore PP, Danielson M, et al. Update: characteristics of a nationwide outbreak of E-cigarette, or vaping, product use-associated lung injury - United States, August 2019-January 2020. MMWR Morb Mortal Wkly Rep. 2020;69(3):90-94.

7. Baughman RP, Culver DA, Judson MA. A concise review of pulmonary sarcoidosis. Am J Respir Crit Care Med. 2011;183(5):573-581.

8. Ohshimo S, Guzman J, Costabel U, Bonella F. Differential diagnosis of granulomatous lung disease: clues and pitfalls: Number 4 in the Series "Pathology for the clinician" Edited by Peter Dorfmuller and Alberto Cavazza. Eur Respir Rev. 2017;26(145):170012.

9. Morris MA, Paudel R, Khosravi M. Vaping induced sarcoidosis flare-up. American Journal of Respiratory and Critical Care Medicine. 2020:201:A6688.

10. Sikka G, Oluyinka M, Schreiber R, Galiatsatos P. Electronic cigarette cessation in youth and young adults: a case series. Tob Use Insights. 2021;14:1179173X211026676.

11. Nunes H, Uzunhan Y, Gille T, Lamberto C, Valeyre D, Brillet PY. Imaging of sarcoidosis of the airways and lung parenchyma and correlation with lung function. Eur Respir J. 2012;40(3):750-765.

12. Hendricks MV, Crosby JH, Davis WB. Bronchoalveolar lavage fluid granulomas in a case of severe sarcoidosis. Am J Respir Crit Care Med. 1999;160(2):730-731.

13. Drent M, Crouser ED, Grunewald J. Challenges of sarcoidosis and its management. N Engl J Med. 2021;385(11):1018-1032.

14. Mathew S, Bauer KL, Fischoeder A, Bhardwaj N, Oliver SJ. The anergic state in sarcoidosis is associated with diminished dendritic cell function. J Immunol. 2008;181(1):746-755.

15. Lioy PJ, Weisel CP, Millette JR, Eisenreich S, Vallero D, Offenberg J, Buckley B, et al. Characterization of the dust/smoke aerosol that settled east of the World Trade Center (WTC) in lower Manhattan after the collapse of the WTC 11 September 2001. Environ Health Perspect. 2002;110(7):703-714.

16. Gupta D, Agarwal R, Aggarwal AN, Jindal SK. Molecular evidence for the role of mycobacteria in sarcoidosis: a meta-analysis. Eur Respir J. 2007;30(3):508-516. 